\title{
Physical Parameter Identification of Nonlinear Base-Isolated Buildings Using Seismic Response Data
}

\author{
Chao $\mathrm{Xu}^{1, \dagger}$, J. Geoffrey Chase ${ }^{2}$ and Geoffrey W. Rodgers ${ }^{2}$ \\ ${ }^{1}$ School of Astronautics, Northwestern Polytechnical University, Xi'an 710072, China \\ ${ }^{2}$ Department of Mechanical Engineering, University of Canterbury, Private Bag 4800, Christchurch, New Zealand
}

\begin{abstract}
Base isolation is an increasingly applied earthquake-resistant design technique in highly seismic areas. Examination of the actual performance of isolated structures in real earthquake has become a critical issue. In this paper, a new computational method for system identification is proposed for obtaining insight into the linear and nonlinear structural properties of based-isolated buildings. A bilinear hysteresis model is used to model the isolation system and the superstructure is assumed linear. The method is based on linear and nonlinear regression analysis techniques. Response time histories are divided into different loading or unloading segments. A one-step multiple linear regression is implemented to simultaneously estimate storey stiffness and damping parameters of the superstructure. A two-step regression-based procedure is proposed to identify the nonlinear physical parameters of the isolation system. First, standard multiple linear regression is implemented to deduce equivalent linear system parameters. Analysis of the varying equivalent linear system parameters with displacement is used to distinguish linear and nonlinear segments. Second, nonlinear regression is applied for the nonlinear segments to obtain nonlinear physical parameters. A 3-storey base-isolated building was simulated to real earthquake ground motions and recorded responses were used to demonstrate the feasibility of the proposed
\end{abstract}

\footnotetext{
${ }^{\dagger}$ Corresponding author. Tel.: +86 02988493620

E-mail address: chao_xu@nwpu.edu.cn (C. Xu),geoff.chase@canterbury.ac.nz (J. G. Chase), geoff.rodgers@canterbury.ac.nz (G.W. Rodgers).
} 
method. Superstructure and isolation bearing properties were identified to within $6 \%$ those of actual model value even with a SNR 30dB signal noise level. The overall method allows the simple, effective analysis of nonlinear base isolated structures. The approach to multi-degree of freedom nonlinear structures could be readily generalised to nonlinear, fixed-base, multistorey structures.

KEY WORDS: system identification; multiple linear regression; multi-phase regression; hysteresis; seismic isolation 


\section{INTRODUCTION}

The goal of seismic isolation is to shift the fundamental natural period of a building away from the dominant frequency components of the ground motion. Isolators are placed at the base of the structure to physically decouple it from the foundation and provide flexibility and energy dissipation capability. Base isolation systems have been effective in protecting structures from strong motion earthquakes and are used with increasing popularity [1-4]. However, their effectiveness is dependent on isolator performance. If isolators degrade or fail due to aging, temperature cycles or exceeding their design capacity during extreme events, structural safety is no longer guaranteed. In another case, the isolators were too stiff and no isolation was provided, leading to structural damage [5]. Therefore, the identification and monitoring of the building isolation performance is increasingly important in civil engineering.

Recorded seismic responses contain a lot of information about the dynamic properties of the structures and isolators. A number of studies to identify the actual performance of the baseisolated structures subjected to seismic excitation have been conducted. The four baseisolated buildings affected by the 1994 Northridge earthquake were identified as equivalent linear dynamic systems characterized by time-invariant or time-variant modal parameters in [6]. Nagarajaiath and Sun [7] investigated the seismic response and performance evaluation of the base-isolated USC Hospital Building. Both parametric and non-parametric system identification methods were applied to estimate the equivalent linear system periods and damping. The same method was then used to identify the base-isolated Los Angeles Country Fire Command and Control building in [8]. In a later work, a time segmented least squares technique was proposed for the building and time-variant linear system properties were 
obtained in [9]. Recently, Yoshimoto et al [10] proposed a multiple-input multiple-output (MIMO) subspace identification method for damage detection of the base-isolated buildings. Time piecewise system parameters, such as the lateral stiffness and damping coefficient, were identified as damage indices. Loh et al. [11] developed a recursive subspace identification method to identify the time-variant periods and damping of the mid-story isolation buildings using earthquake records. Yao and Pakzad [12] proposed time and frequency domain linear regression methods to identify interstory stiffness of a shear frame structure subjected to white noise excitation.

However, these studies are all based on an assumed linear equivalent system over the whole time history or piecewise linear systems over different time segments. Nonlinear physical parameters of the structural system, which are more attractive for understanding the actual dynamic characteristics of base-isolated buildings, and which are critical to structural control and health monitoring, cannot be directly obtained. Equally, it is these parameters, linear and nonlinear, which are used to specify isolators and in design. Thus, directly identifying these parameters would best suit designers and practitioners.

A limited number of methods addressed nonlinear physical parameter identification of baseisolated structures, have been developed. Tan and Huang [13] proposed an iterative trial and error optimization procedure to identify the physical parameters of the linear superstructure and bilinear hysteretic isolators. The essence of the study is the application of a Masing criterion to transform the multi-value hysteretic restoring force into a single-value function such that ordinary identification processes can be used. The method needs complex iterations and is computationally costly. Similar identification methods were then extended to different base-isolated structures in [14-17]. Furukawa et al. [18] proposed a least squares prediction- 
error minimization method to identify a base-isolated building in Kobe City affected by the 1995 Hyogoken-Nambu earthquake. The isolation system was identified based on three different models: a linear equivalent model, a bilinear model and a tri-linear model. Results show that the nonlinear model parameters can be reasonably estimated and the tri-linear model best fit the actual isolator hysteretic behaviour and response time histories. Ahn and Chen [19] proposed a nonlinear model-based system identification method for a three-span continuous base-isolated bridge. It used the Mengotto-Pinto model to model hysteresis behaviour of the lead-rubber bearings. Nonlinear model parameters were pulled out by a two phase output-error optimization algorithm to address ill-conditioning issues. Xie and Mita [20] presented a method to estimate the restoring force of an isolation layer using component mode synthesis (CMS). The amplitude-dependent equivalent system stiffness and damping coefficients were identified to characterize the nonlinearity of the isolation layer. Oliveto et al. [21] developed a time domain nonlinear system identification procedure to determine the physical parameters of the hybrid seismic isolation system of a base-isolated building. The method used a bilinear hysteretic model and a constant coulomb friction model to model the high damping rubber bearings and low friction sliding bearing respectively. The model parameters were estimated by a nonlinear least squares output-error minimization method using free vibration test data. The Covariance Matrix Adaptation- Evolution Strategy (CMSES) algorithm was proposed for identification of nonlinear base isolation system from earthquake records in [22].

Hence, nonlinear isolation system parameters can be identified. However, most of these methods belong to classes of output-error algorithms that depend on a specific mechanics model, which may or may not fully match the measured response due to material nonlinearities, degradation or variability in construction. Furthermore, these methods are 
applied after the whole time history data are obtained and cannot be applied in real-time, near real-time, or in situ. In particular, an optimization iteration algorithm is needed to derive model parameters, as well as added algorithm control parameters that are often manually adjusted to get a good result given the complex optimization algorithm applied. Thus, there is a major need for a much simpler, more efficient approach to capturing nonlinear behaviours that is amenable to real-time or near real-time results and requires no specific model and less operator input.

It should also be noted that structural control arena there have been some simpler real-time approaches. In particular most have dealt with strictly linear structure stiffness or/and modal parameters [23-25], which is not the case here. Among relatively simpler nonlinear structural identification methods in the control arena, Wu and Smyth [26] used a non-parametric unscented Kalman filter to identify five of fourteen parameters in one generalised hysteretic model with minimal noise, while Smyth et al [27] used a similar adaptive filtering approach for a Bouc-Wen modeled hysteretic structure. All of these are mechanics model dependent and identify some parameters that would allow a nonlinear elasto-plastic stiffness to be identified for a record, but not cycle to cycle.

In this paper, a new computational method for identification of physical parameters of nonlinear base-isolated buildings is developed and validated. The algorithm is based on linear and nonlinear statistical regression analysis techniques. It yields nonlinear physical parameters of the isolation system, as well as linear stiffness and damping parameters of the isolated structure. The proposed method can be generalised to various forms of nonlinearity in the isolation layer. A proof-of-concept case study was performed to demonstrate and prove the method. 


\section{STRUCTURAL MODEL}

Consider the base-isolated $N$-storey shear building in Figure 1 . Let $m_{i}, k_{i}, c_{i}$ denote the mass of the $i$-th level, the stiffness and the viscous damping coefficient of the $i$-th storey, respectively. Let $u_{i}$ denote the displacement of the $i$-th ceiling in relation to the ground. The base mass is $m_{0}$ and relative displacement is $u_{0}$. A typical base-isolated building can be separated into two structural systems: a superstructure and a base isolation layer. Various base isolation systems have been extensively developed in recent years [1, 28, 29]. The lead rubber bearing (LRB) has been the most popular base isolation system among others for practical implementation in New Zealand, Japan, the United States, China, and other seismic areas. Without the loss of generality, the base isolated building is assumed equipping with LRBs and additional viscous dampers which help to maximize energy absorption. The response of the upper superstructure can be effectively reduced to an acceptable level through the installation of the base isolation system. Thus, It is reasonable that the superstructure is assumed remaining within the elastic range during an earthquake and the nonlinearity is only associated with the base-isolated systems. 


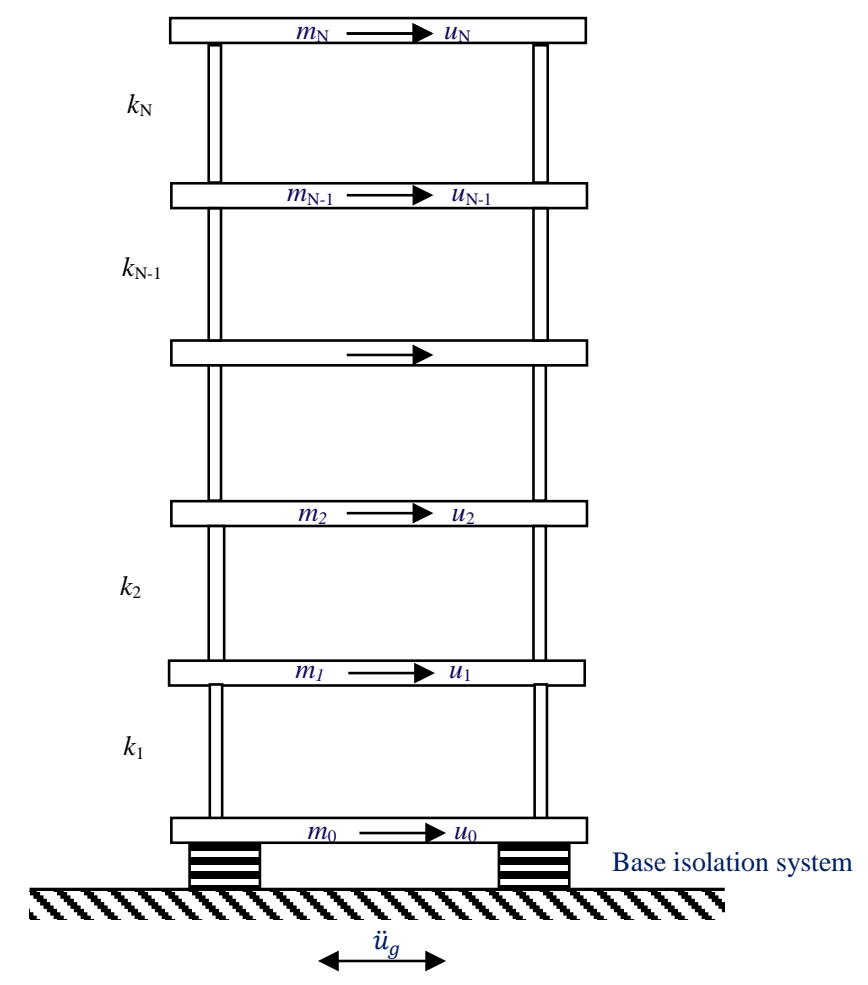

Figure $1 \mathrm{~N}$-storey base-isolated shear building model

The isolation system can be characterized by a hysteretic restoring force $f_{r}$ and a viscous damper coefficient $c_{0}$. The equation of motion for the base-isolated multi-storey building subjected to ground acceleration $\ddot{u}_{g}$ can then be expressed:

$$
M \ddot{u}+C \dot{u}+K \boldsymbol{u}+\boldsymbol{R}=-\boldsymbol{M} I \ddot{u}_{g}
$$

in which $\boldsymbol{u}=\left\{\begin{array}{llll}u_{0} & u_{1} & \cdots & u_{N}\end{array}\right\}^{T}$ and the structural mass matrix $\boldsymbol{M}$, damping matrix $\boldsymbol{C}$, linear stiffness matrix $\boldsymbol{K}$, isolators restoring force matrix $\boldsymbol{R}$ and unity vector $\boldsymbol{I}$ are defined:

$$
\boldsymbol{M}=\left[\begin{array}{llll}
m_{0} & & & 0 \\
& m_{1} & & \\
& & \ddots & \\
0 & & & m_{N}
\end{array}\right]
$$




$$
\begin{aligned}
& \boldsymbol{C}=\left[\begin{array}{cccccc}
c_{0}+c_{1} & -c_{1} & & & & \mathbf{0} \\
-c_{1} & c_{1}+c_{2} & & & & \\
& & \ddots & \ddots & & \\
& & \ddots & \ddots & & \\
0 & & & & c_{N-1}+c_{N} & -c_{N} \\
\mathbf{0} & & & & -c_{N} & c_{N}
\end{array}\right] \\
& \boldsymbol{K}=\left[\begin{array}{cccccc}
k_{1} & -k_{1} & & & & \mathbf{0} \\
-k_{1} & k_{1}+k_{2} & & & & \\
& & \ddots & \ddots & & \\
& & \ddots & \ddots & & \\
& & & & k_{N-1}+k_{N} & -k_{N} \\
\mathbf{0} & & & & -k_{N} & k_{N}
\end{array}\right] \\
& \boldsymbol{R}=\operatorname{diag}\left\{\begin{array}{llll}
f_{r} & 0 & \ldots & 0
\end{array}\right\} \\
& \boldsymbol{I}=\left\{\begin{array}{llll}
1 & 1 & \cdots & 1
\end{array}\right\}^{T}
\end{aligned}
$$




\section{IDENTIFICATION ALGORITHM AND PROCEDURE}

\subsection{Physical parameter identification of the superstructure}

With a linear model assumption for the superstructure, the equations of motion of the shear

superstructure can be separated from Equation (1) and written in a non-compact form:

$$
\begin{gathered}
m_{N} \ddot{u}_{N}+c_{N}\left(\dot{u}_{N}-\dot{u}_{N-1}\right)+k_{N}\left(u_{N}-u_{N-1}\right)=-m_{N} \ddot{u}_{g} \\
m_{i} \ddot{u}_{i}+c_{i}\left(\dot{u}_{i}-\dot{u}_{i-1}\right)+k_{i}\left(u_{i}-u_{i-1}\right)-c_{i+1}\left(\dot{u}_{i+1}-\dot{u}_{i}\right)-k_{i+1}\left(u_{i+1}-u_{i}\right)=-m_{i} \ddot{u}_{g} \\
i=1 \sim N-1
\end{gathered}
$$

A revised equation of motion for the $i$-th storey can be derived by summing Equation (3) from $i$ to $N$, and is defined:

$$
-\sum_{j=i}^{N} m_{j}\left(\ddot{u}_{g}+\ddot{u}_{j}\right)=c_{i}\left(\dot{u}_{i}-\dot{u}_{i-1}\right)+k_{i}\left(u_{i}-u_{i-1}\right) \quad i=1 \sim N
$$

The response variables at each measurement instant $k$ can then be defined:

$$
\begin{gathered}
y_{k}=-\sum_{j=i}^{N} m_{j}\left(\ddot{u}_{g, k}+\ddot{u}_{j, k}\right) \\
x_{1, k}=\dot{u}_{i, k}-\dot{u}_{i-1, k} \\
x_{2, k}=u_{i, k}-u_{i-1, k}
\end{gathered}
$$

Note that these variables can be obtained directly or indirectly from measurement and the prior known mass data. Equation (4) should hold for these response variables at each instant. 
Thus, the problem of physical parameter identification of the linear superstructure is transformed to a search for the linear relationship between the variables $y_{k}$ and $x_{1, k}, x_{2, k}$. If the response variables at each instant are considered as an observation pair, the identification problem can be represented by a general multiple linear regression model [30]:

$$
\begin{gathered}
\boldsymbol{Y}=\boldsymbol{X} \boldsymbol{\beta}+\boldsymbol{\epsilon} \\
\boldsymbol{Y}=\left\{\begin{array}{c}
y_{1} \\
y_{2} \\
\vdots \\
y_{k} \\
\vdots \\
y_{n}
\end{array}\right\}, \quad \boldsymbol{X}=\left[\begin{array}{ccc}
1 & x_{1,1} & x_{2,1} \\
1 & x_{1,2} & x_{2,2} \\
\vdots & \vdots & \vdots \\
1 & x_{1, k} & x_{2, k} \\
\vdots & \vdots & \vdots \\
1 & x_{n, k} & x_{n, k}
\end{array}\right], \quad \boldsymbol{\beta}=\left\{\begin{array}{l}
\beta_{0} \\
\beta_{1} \\
\beta_{2}
\end{array}\right\}, \quad \boldsymbol{\epsilon}=\left\{\begin{array}{c}
\epsilon_{1} \\
\epsilon_{2} \\
\vdots \\
\epsilon_{k} \\
\vdots \\
\epsilon_{n}
\end{array}\right\}
\end{gathered}
$$

where $n$ is the number of all observed response variable pairs, $\boldsymbol{Y}$ is regressand, $\boldsymbol{X}$ is the repressor, $\boldsymbol{\beta}$ is the regression coefficients vector to be estimated, and $\boldsymbol{\epsilon}$ is the vector of estimation error at different times and is random and normally distributed. Note that $\boldsymbol{\epsilon}$ represents the inevitable measurement noise and model uncertainty in this regression analysis.

The least squares principle can then be used to find the unbiased estimates of the regression coefficients by choosing the regression line that is the most "closest" line to all data points $\left(y_{k}, x_{1, k}, x_{2, k}\right)$, i.e.,

$$
\left(b_{0}, b_{1}, b_{2}\right)^{T}=\arg \min _{\boldsymbol{\beta}}\left[(\boldsymbol{Y}-\boldsymbol{X} \boldsymbol{\beta})^{\prime}(\boldsymbol{Y}-\boldsymbol{X} \boldsymbol{\beta})\right]
$$

where $\operatorname{argmin}[$.$] stands for the argument of the minimum, and the vector \left(b_{0}, b_{1}, b_{2}\right)^{T}$ is the least squares estimates of the regression coefficients vector. Comparing Equation (6) and Equation (4), it is clear that the resulting regression coefficient $\beta_{1}$ represents the relationship 
between velocity and restoring force, and the coefficient $\beta_{2}$ represents the relationship between displacement and restoring force. Thus, $b 1$ is the estimate of damping parameter and $b 2$ is the estimate of stiffness parameter in Equation (4).

\subsection{Physical parameter identification of the isolation layer}

The equation of motion for the isolation layer is defined:

$$
-\sum_{j=0}^{N} m_{j}\left(\ddot{u}_{g}+\ddot{u}_{j}\right)=c_{0} \dot{u}_{0}+f_{r}
$$

Note that the restoring force of the base isolator is highly nonlinear and dependent on the displacement history. It is difficult to accurately describe the nonlinear force-deformation behaviour of the isolator. However, according to experimental observation, the inelastic response of LRB isolators can be effectively modelled using a bilinear hysteretic law [31]. The restoring force-deformation relationship of the isolator is thus defined:

$$
f_{r}\left(u_{0}\right)=f_{r}\left(u_{0}^{I}\right)+2 g\left(\frac{u_{0}-u_{0}^{I}}{2}\right)
$$

where $I$ is the instant of most recent loading reversal and $u_{0}^{I}$ is the base relative displacement at instant $I$. The bilinear backbone curve function $g$ is defined [16]:

$$
g(v)=\left\{\begin{array}{cc}
k_{e} v & -d_{y} \leq v \leq d_{y} \\
k_{e} d_{y}+k_{p}\left(v-d_{y}\right) & v>d_{y} \\
-k_{e} d_{y}+k_{p}\left(v+d_{y}\right) & v<-d_{y}
\end{array}\right.
$$


where $v$ is the displacement, $d_{y}$ denotes the yielding displacement, and $k_{e}$ and $k_{p}$ are elastic stiffness and post-yielding stiffness, respectively. The later three parameters fully characterize nonlinear properties of the isolator and are the physical parameters to be identified.

The hysteretic restoring force of the isolation system is path dependent and not a single-value function of displacement. It is dependent on deformation history and on whether the deformation is increasing (positive velocity) or decreasing (negative velocity). More importantly, the left side of Equation (8) is not linearly related to the variable $u_{0}$. Therefore, the physical parameters of the isolation layer cannot be directly obtained by a standard multiple linear regression analysis. A two-step regression-based procedure is thus developed to solve the problem.

\subsubsection{Partition of the response history}

The isolation system subjected to a strong-motion earthquake may experience several cycles of deformation, showing a general hysteresis history, as illustrated in Figure 2. It can be seen that the nonlinear restoring force is path dependent and multi-valued. However, within a time segment where the velocity holds the same sign, the restoring force is a single-value function of the deformation. Hence, the whole response history can be sliced into many sub-cycles and sub-half cycles according to the points where the velocity is zero.

These points are called turning points. For example, if the turning points $\left(x_{3}, x_{5}\right.$ and $\left.x_{7}\right)$ have been known, the data points between $x_{3}$ and $x_{7}$ form a sub-cycle $i$ that includes a full loading and unloading path. Two sub-half cycles can be obtained through dividing this sub- 
cycle by another turning point $x_{5}$. The point $x_{5}$ is not only the ending point of the loading path, but also the starting point of the very next unloading path in this sub cycle. Obviously, the hysteretic restoring force between two successive points in one half cycle is single-valued and either monotonically increasing or decreasing. Thus, regression analysis can be implemented for these half cycle data separately to obtain the best approximation. The estimated regression coefficients will then relate to the system physical parameters in these half cycles.
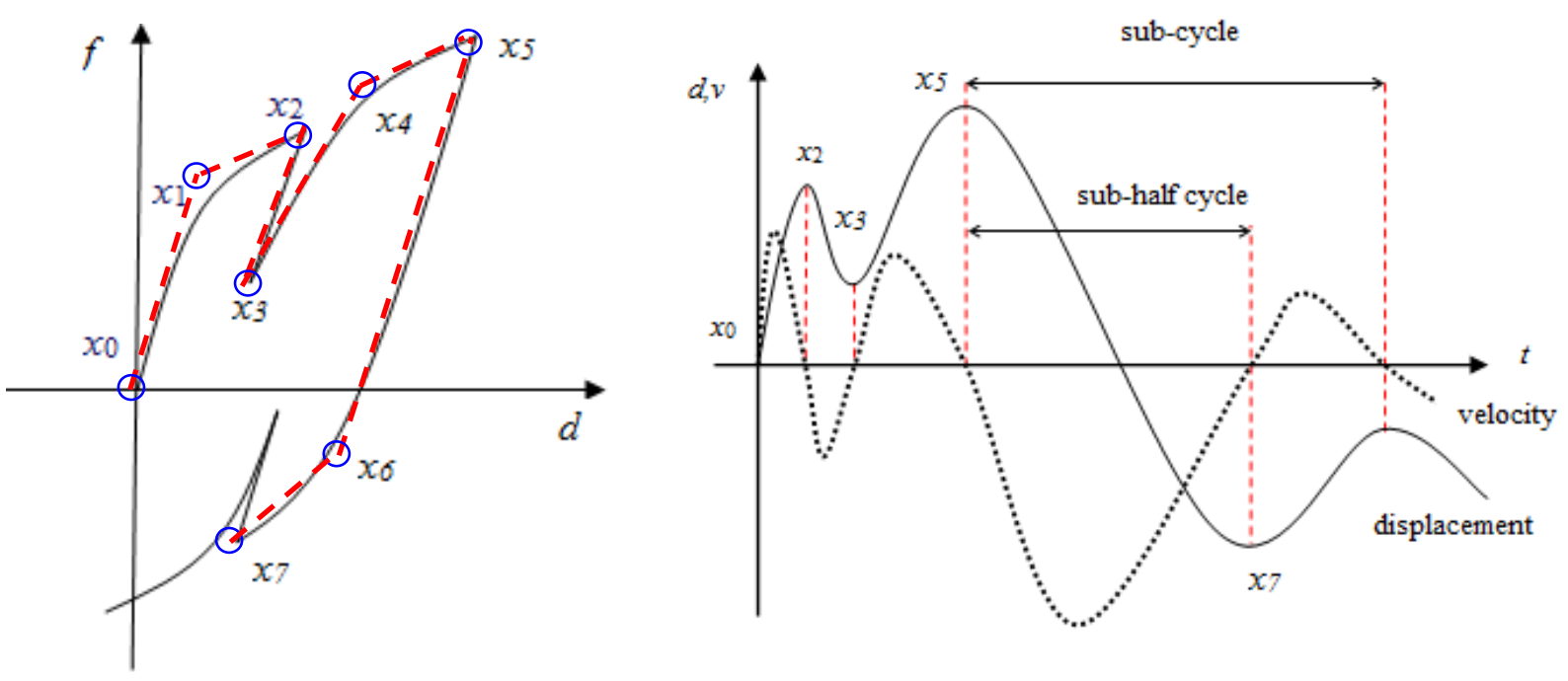

Figure 2 (a) a general hysteresis loop; $\quad$ (b) Sub-cycle and sub-half cycle segments

Generally, during an earthquake event, some half-cycle responses are linear and some nonlinear. To identify the nonlinear model parameters, the first step is to identify and separate the linear and nonlinear half cycles. For linear half cycles, a standard multiple linear regression can be used directly. For nonlinear half cycles, a nonlinear regression technique is needed owing to nonlinear relationship between variables. 


\subsubsection{The first step: standard multiple linear regression to each half cycle}

A standard multiple linear regression is separately implemented to a set of data points in each half cycle first. It actually assumes an equivalent linear system model for each half cycle. Thus, the derived regression coefficients give the estimate of the equivalent linear system stiffness $k_{l}$ and viscous damping coefficient $c_{l}$ within the segment. When there is no inelastic deformation presented in the half-cycle, the equivalent linear system stiffness should approach a realistic system elastic stiffness $k_{e}$. However, when there is inelastic deformation, this equivalent linear system stiffness should capture a secant average stiffness of $k_{e}$ and $k_{p}$, and be notably lower.

The equivalent linear stiffness $k_{l}$ varies over different half-cycle segments. Varying $k_{l}$ is a significant indicator of the nature of the dynamic system. It is reasonable that the hysteresis curve responds linearly where sub-half cycle displacement increment $\Delta d$ is small and nonlinearly where $\Delta d$ is larger than the structural yield displacement. Thus, a rapid drop in $k_{l}$ at large displacement increment can be viewed as a good indicator of inelastic behaviour during that specific half cycle. The analysis of $k_{l}$ versus $\Delta d$ thus gives an indicator to identify potential inelastic sub-half cycles. In addition, the estimated equivalent linear damping coefficient $c_{l}$ is the measure of system energy dissipation. System energy dissipation capacity will similarly increase due to the added hysteretic damping. Thus, the analysis of $c_{l}$ versus $\Delta d$ may also be used as another indicator of the inelastic sub half cycles. Bothe indicators of inelastic behaviour can be automated and easily found using a threshold.

\subsubsection{The second step: nonlinear regression to identified nonlinear sub-half cycles}

Many estimates of viscous damping coefficient $c_{0}$ and elastic stiffness $k_{e}$ can be directly obtained from multiple linear regression analysis of those identified linear half cycles. The 
mean of $c_{0}$ estimates is identified as 'actual' viscous damping coefficients of the isolation layer and will be used as a known parameter at the second identification step. Therefore, Equation (8) can be written:

$$
-\sum_{j=0}^{N} m_{j}\left(\ddot{u}_{g}+\ddot{u}_{j}\right)-c_{0} \dot{u}_{0}=f_{r}
$$

During a loading or unloading inelastic half cycle, the restoring force is a piecewise linear function of displacement with parameters $k_{e}, k_{p}$ and $d_{y} . k_{p}$ is usually only about 5\%-10\% of $k_{e}$ for typical isolators. To identify the final elastic stiffness, $k_{e}$, and post-yielding stiffness, $k_{p}$, simultaneously from Equation (11), data points in the identified nonlinear half cycle must be divided into two segments, and a different linearly parameterized polynomial identified via regression analysis for each segment. The difficulty is associated with the unknown interaction point of each segment and the join point of the segmented regression lines has to be estimated to ensure continuity throughout the nonlinear half cycle.

This situation is a special nonlinear regression problem, named multi-phase regression with unknown transition points. The problem has a long history in mathematics [32-34] and has been applied in some engineering fields [35, 36]. However, according to the author's best knowledge, it has not been used in structural dynamic system identification.

First, define:

$$
\begin{gathered}
y_{k}=-\sum_{j=0}^{N} m_{j}\left(\ddot{u}_{g, k}+\ddot{u}_{j, k}\right)-c_{0} \dot{u}_{0, k} \\
x_{k}=u_{0, k} \quad k=1,2, \ldots, m
\end{gathered}
$$


in which, $m$ is the number of data points in a nonlinear half cycle. Herein, a two-phase linear regression function can be defined:

$$
f(x, \beta)= \begin{cases}\beta_{10}+\beta_{11} x, & x \leq x_{0} \\ \beta_{20}+\beta_{21} x, & x>x_{0}\end{cases}
$$

where $\alpha=\left\{\beta_{10}, \beta_{11}, \beta_{20}, \beta_{21}\right\}^{\mathrm{T}}$ is the set of the unknown regression coefficients of each segment and $x_{0}$ is the unknown join point. The join point satisfies the linear constraint to ensure the continuity of the solution at the interaction point:

$$
\beta_{10}+\beta_{11} x_{0}=\beta_{20}+\beta_{21} x_{0}
$$

Using the least-squares estimation, the regression analysis seeks the best estimate of the vector $\boldsymbol{\alpha}$ that minimizes the residual sum:

$$
R(\boldsymbol{\alpha})=\sum_{k=1}^{m}\left[y_{k}-f\left(x_{k}, \beta\right)\right]^{2}
$$

In case of the function (13), the sum $R(\boldsymbol{\alpha})$ can be expressed as

$$
R(\boldsymbol{\alpha})=\sum_{x_{k} \leq x_{0}}\left[y_{k}-\left(\beta_{10}+\beta_{11} x_{k}\right)\right]^{2}+\sum_{x_{k}>x_{0}}\left[y_{k}-\left(\beta_{20}+\beta_{21} x_{k}\right)\right]^{2}
$$

where the solution of Equation (16) is subject to the constraint Equation (14).

To minimize the function $R(\boldsymbol{\alpha})$, a method similar to [35] is applied. Conceptually, if the transition point is known, the minimum of $R(\boldsymbol{\alpha})$ can be found by computing a standard linear 
regression for each segment. Thus, given an arbitrary data partition between point $I$ and $I+1$, the residual sum can be minimized over $\boldsymbol{\alpha}_{\boldsymbol{I}}=\left\{\beta_{10}, \beta_{11}, \beta_{20}, \beta_{21}\right\}^{T}$, and this outcome yields a sequence of residual sum function $R_{I}(\boldsymbol{\alpha})(I=2, \ldots, m-2$, ) . The goal is to pick the $I$ that gives the minimum value for $R_{I}(\boldsymbol{\alpha})$. Note that this is true only when $x_{I} \leq x_{0} \leq x_{I+1}$. Thus, the estimation of $x_{0}$ has to be computed using the constraint Equation (14) from the element of $\boldsymbol{\alpha}_{\boldsymbol{I}}$ to check that $x_{0}$ is in fact between the two data points $I$ and $I+1$. If so, the final solution is found. If it is not, a confidence interval method presented in [35] can be used to check whether to attribute this issue to observation outliers or an incorrect assumption.

It can be seen from Equation (13) that the resulting regression coefficients, $\beta_{11}$ and $\beta_{21}$, represent the relationship between displacement and restoring force. They correspond the elastic stiffness term, $k_{\mathrm{e}}$, and post-yielding stiffness term, $k_{\mathrm{p}}$, in Equation (10), respectively. The estimated joint point is the elastic/inelastic response turning point within the nonlinear half cycle. It is closely related to the isolation system yield displacement $d_{y}$. For an unloading nonlinear half cycle $i, d_{y}$ can be defined:

$$
d_{y i}=\frac{x_{\operatorname{maxi}}-x_{0 i}}{2}
$$

where $x_{\text {maxi }}$ is the displacement at the instant of most recent loading reversal; and $x_{0 \mathrm{i}}$ is estimated join point. For a loading nonlinear half cycle $i$ :

$$
d_{y i}=\frac{x_{0 i}-x_{\min i}}{2}
$$

where $x_{\operatorname{mini}}$ is at the instant of most recent loading reversal. 
Through the proposed nonlinear regression procedure, many estimates of the elastic and postyielding stiffness, and yield displacement are obtained. The last output of each parameter is calculated by averaging of these estimated values over the time history. The flowchart of the identification method proposed is illustrated in Figure 3.

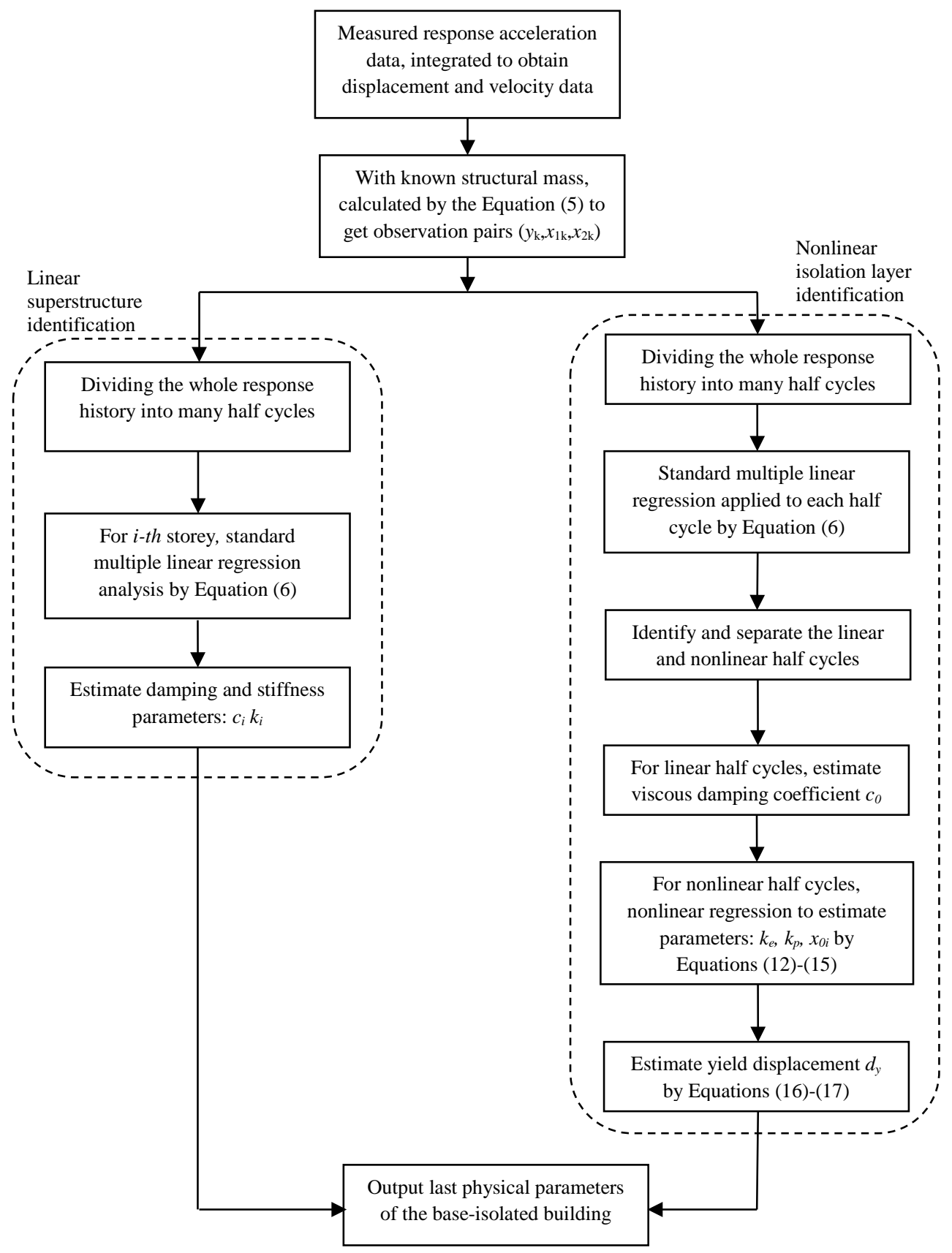

Figure 3 The flowchart of the identification procedure for base-isolated buildings 


\section{PROOF-OF-CONCEPT CASE STUDY}

The proof-of-concept case used in this investigation is the three-story base-isolated shear building studied by Huang et al [16]. The floor masses, storey stiffnesses, storey viscous damping coefficients and nonlinear model parameters of the isolation system are shown in Figure 4. Nonlinear responses of the multi-storey base-isolated building to a real earthquake motion are simulated using the Newmark's step-by-step method of integration. The selected ground motion is the record at the Coyote Lake Dam station during the 1989 earthquake event in Loma Prieta, California. The duration of the ground excitation is approximately 40s with a peak ground acceleration (PGA) of $0.484 \mathrm{~g}$. The ground acceleration record is available at a sample time step of $0.005 \mathrm{~s}$ and filtered with low-pass and high-pass cut-off frequencies of 33 and $0.1 \mathrm{~Hz}$, respectively, shown in figure 5.
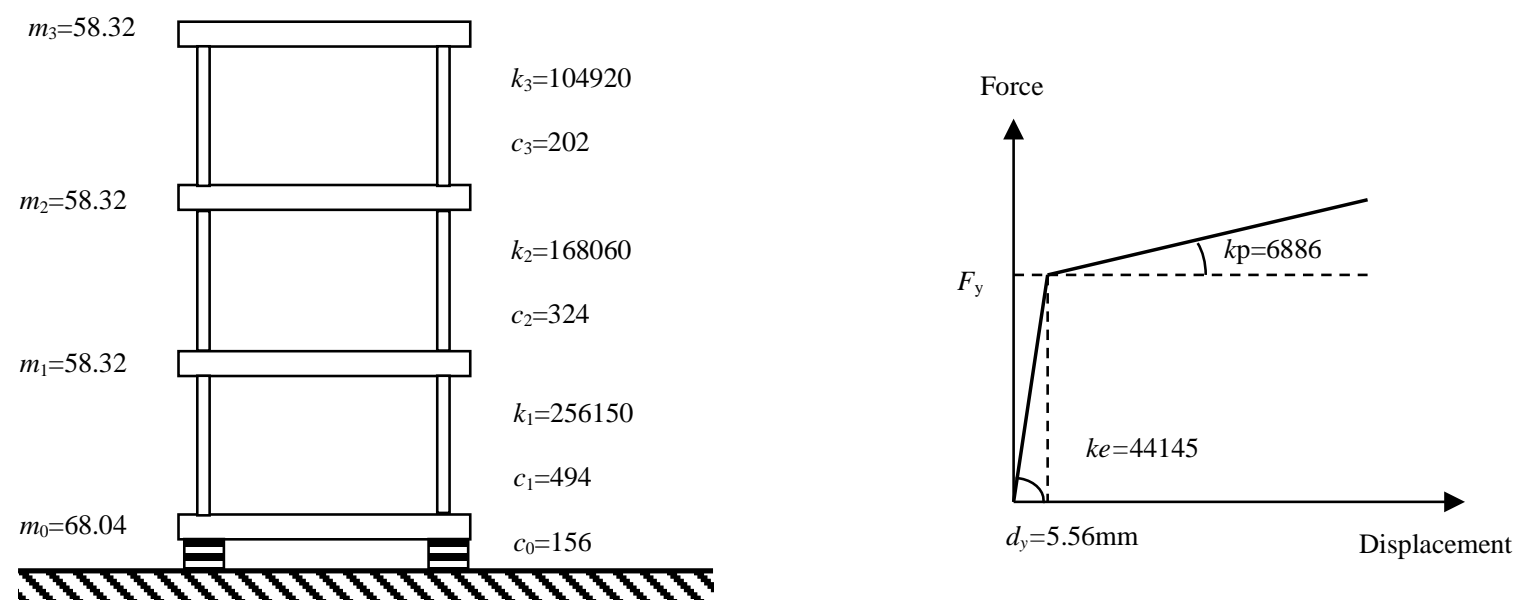

Figure 4 Model parameters of the 3-storey base-isolated building; (The unit for stiffness is N/mm, for damping coefficient is N.sec/mm, and for mass is ton)

The time histories of relative displacement at each floor are shown in Figure 6. By comparing the time histories of the top and base floors, one can realized that they are similar both in 
magnitude and the phase. Therefore, the upper structure can be modelled as a linear superstructure because interstory drifts in superstructure are very small.

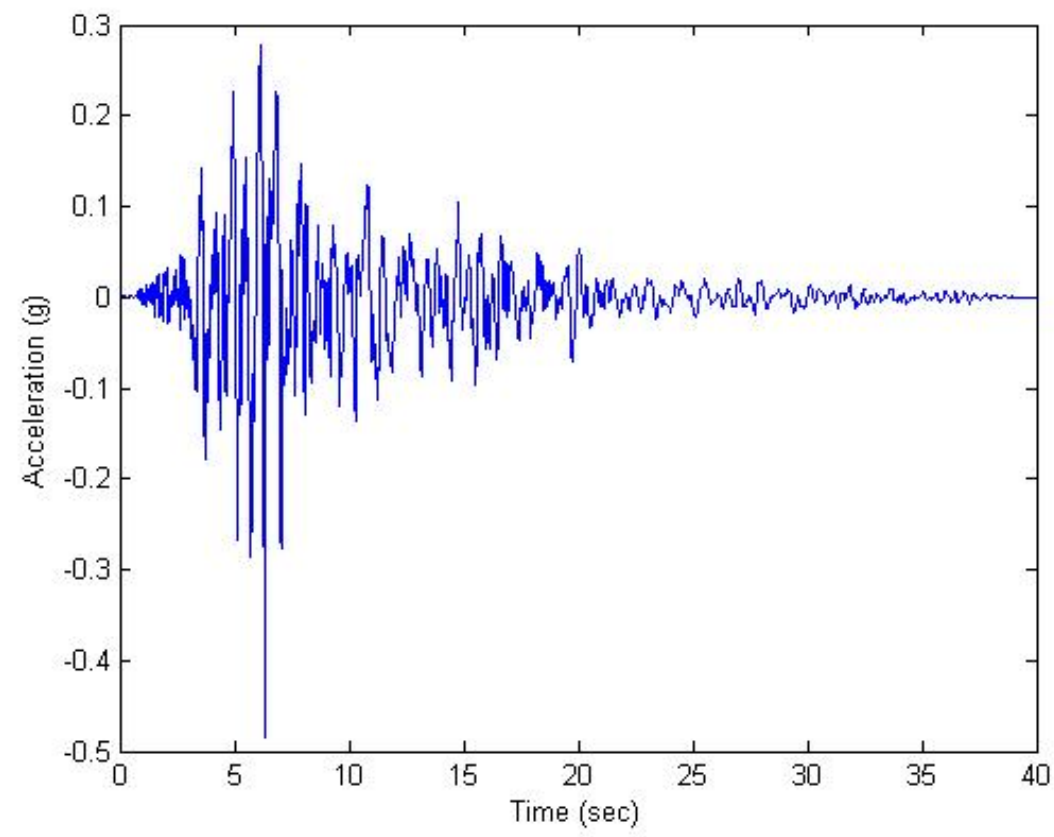

Figure 5 Ground acceleration recorded at the Coyote Lake Dam station during the 1989 Loma Prieta event

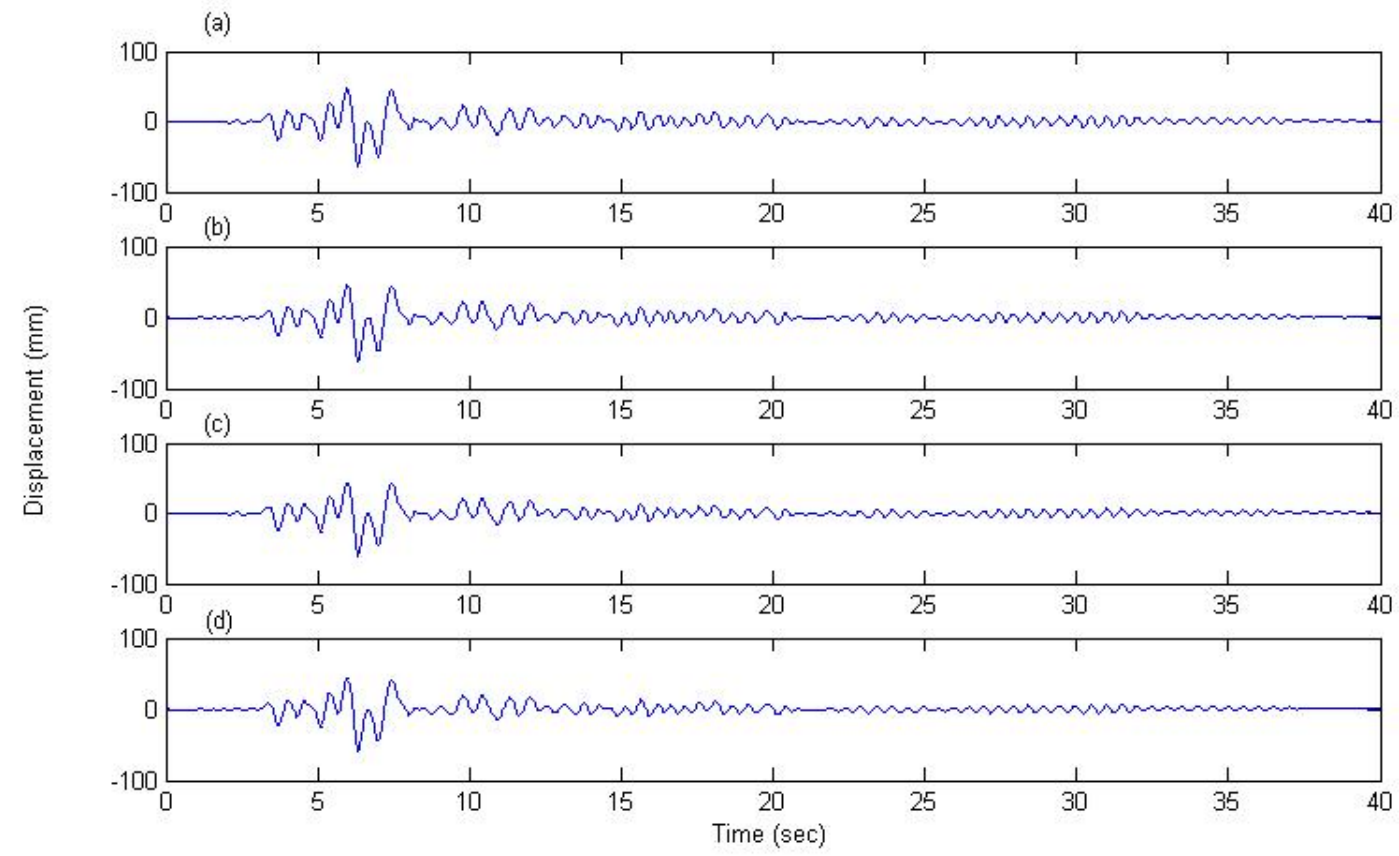

Figure 6 Time histories of relative displacement: (a) top floor; (b) second floor; (c) first floor; (d) base floor 
Figure 7 presents the total restoring force-displacement hysteretic loop of the base isolation layer. It can be seen that the isolation system experiences several inelastic hysteresis cycles. The isolation bearings show a ductility ratio of 8.232, which dissipates most of the energy of the ground inputs such that the superstructure interstory drifts are much reduced.

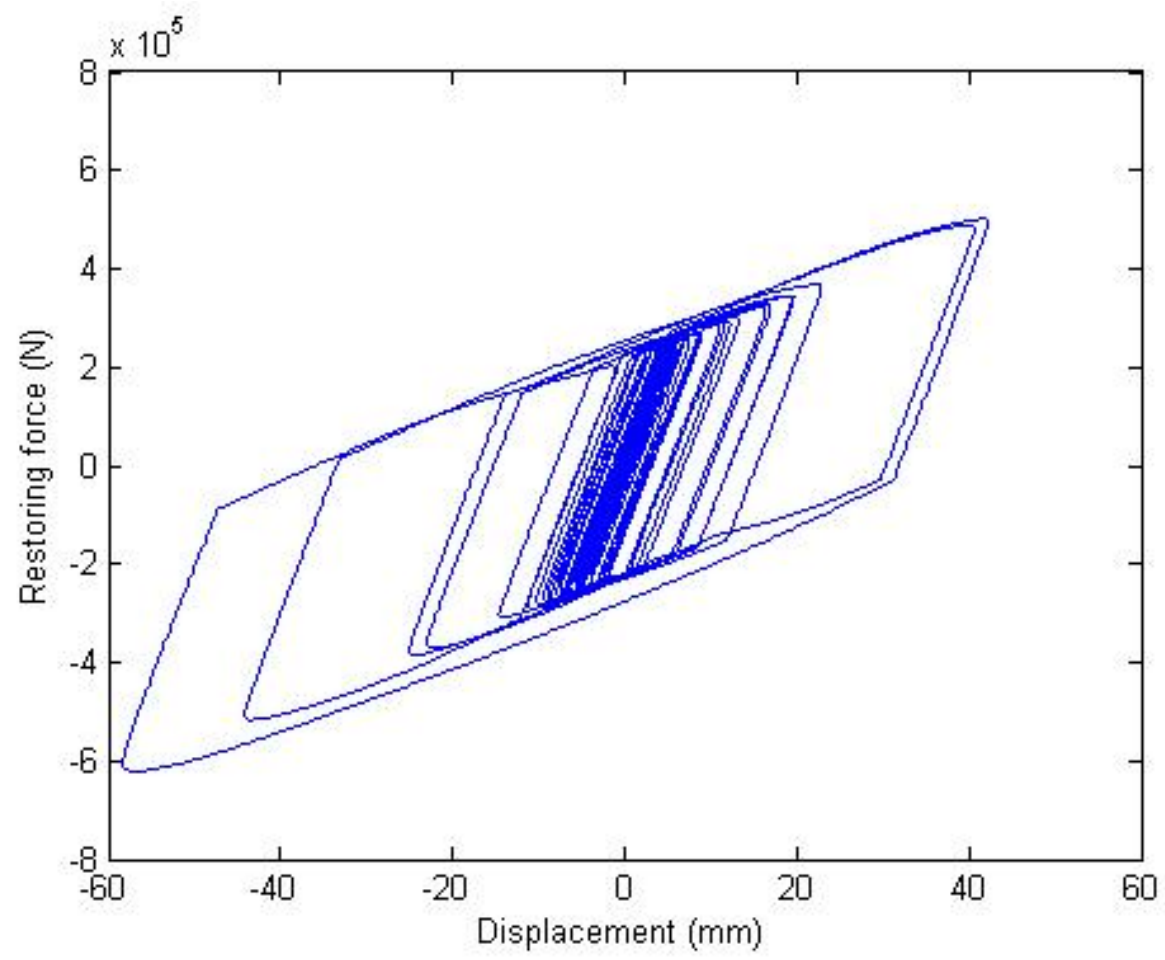

Figure 7 Restoring force-displacement hysteresis loop of the isolation system

It is noted that the free-field ground motion is used as the system input in this proof-ofconcept study. In real world application, the actual earthquake input to the building is not accurately known as the effect of soli-structure coupling. However, this limitation can be overcome by placing an accelerometer on the foundation. The records on the foundation can be used as earthquake input to the building.

For system parameter identification, displacement and velocity histories are estimated from numerical integration of acceleration history. Numerical integration is sensitive to noise and 
thus subject to drift and numerical error. In practice, the recommended mean removal and band-pass filters will not always produce satisfactory results, particularly if permanent deformation occurs [37]. However, additional sensors are increasingly used in civil engineering, and integration errors can be effectively corrected by data fusion of a wide range of different sensors [38-41]. The low-solution-measured displacement corrected acceleration integration method proposed by Hann et al [39] was applied to get the displacement and velocity estimates. In this case study, the low-solution-measured displacement was taken at $1 \mathrm{~Hz}$ and assumed to be a 1000pt backward moving average of $1000 \mathrm{~Hz}$ calculated displacement data available from simulation. Acceleration data was taken at $1000 \mathrm{~Hz}$.

Sensor noise was added to realistically test robustness of the method. A separate white noise corresponding to different signal-noise-ratios (SNR) was added to the simulated noise-free acceleration and displacement measurements, respectively, to mimic a realistic situation over a range of possible sensor performance. All these responses and prior known mass data were used as inputs to the identification procedure. The physical parameters of each storey are identified floor-to-floor. The identification procedure with random added noise was run 100 times to generate final statistical results for each noise level. 


\section{RESULTS AND DISCUSSIONS}

\subsection{Physical parameter identification of the superstructure}

The response time histories are first sliced into many segments. Each segment is a monotonic loading or unloading half cycle. Standard multiple linear regression analysis was implemented to those half cycles and many estimates of interstory stiffness and damping parameters were obtained. The plots of estimated physical parameters for the superstructure at different half-cycle displacement increments using noise-free signals are shown in Figure 8.

It can be seen from Figure 8 that the estimates of stiffness and damping parameter vary very slightly around a linear constant except at very small half cycle displacements. These results show that the superstructure is indeed identified as linear during the earthquake. The accuracy of regression analysis is closely related to the number and distribution of observed sample points. At the segments with very small displacement increments, the time duration is very short and the observed samples are thus very limited and concentrated with the resulting impact on estimated parameters. It is reasonable to discard these few outlying identification results. Therefore, only these segments with displacement increment exceeding $1 \mathrm{~mm}$ are considered to estimate the final storey stiffness and damping.

The statistical results of identified stiffness and damping parameters from 100 runs for each noise level are summarized in Table 1. The proposed identification method yields good estimates of the storey stiffness and damping parameters even using signals with significant added random noise. The stiffness identification results are more accurate than the damping results, which is desirable. As the noise level increases, the identified parameter mean error and standard deviation both increase. However, as long as the SNR is larger than $30 \mathrm{~dB}$, the 
estimated means of stiffness and damping parameters match very well with the actual values. Thus, information in Table 1 indicates that the proposed time-segmented multiple linear regression method for the superstructure yields good accuracy to within $2.47 \%$ for stiffness and $5.71 \%$ for damping (the worst case).
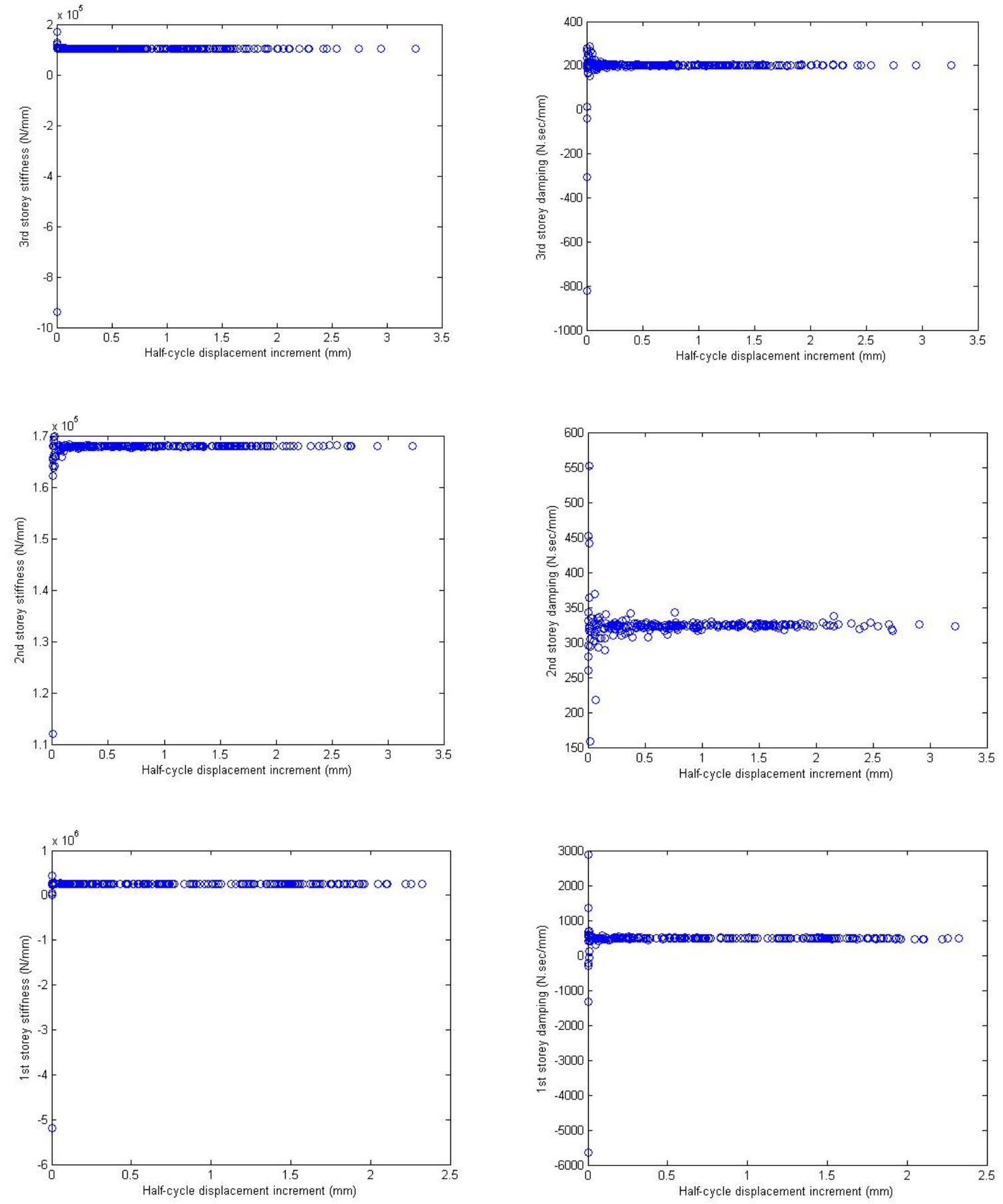

Figure 8 Estimated stiffness and damping paramters for the superstructure using noise-free signals 
Table 1 Identification results for the superstructure

\begin{tabular}{|c|c|c|c|c|c|c|}
\hline & \multicolumn{2}{|c|}{ Storey 3} & \multicolumn{2}{|c|}{ Storey 2} & \multicolumn{2}{|c|}{ Storey 1} \\
\hline & $k_{3}$ & $c_{3}$ & $k_{2}$ & $c_{2}$ & $k_{1}$ & $v_{1}$ \\
\hline \multicolumn{7}{|c|}{ Estimates from noise-free signal } \\
\hline Estimated mean & 104883.25 & 202.00 & 168023.44 & 324.43 & 256016.02 & 488.34 \\
\hline \multicolumn{7}{|c|}{$\begin{array}{l}\text { Estimates from signal } \\
\text { at SNR of } 70 \mathrm{~dB}\end{array}$} \\
\hline Estimated mean & 104881.50 & 202.00 & 168023.84 & 324.44 & 256015.26 & 488.40 \\
\hline Standard deviation & 4.48 & 0.21 & 7.56 & 0.29 & 12.94 & 0.67 \\
\hline \multicolumn{7}{|c|}{$\begin{array}{l}\text { Estimates from signal } \\
\text { at SNR of } 50 \mathrm{~dB}\end{array}$} \\
\hline Estimated mean & 104875.15 & 202.31 & 167997.38 & 324.14 & 255998.55 & 488.40 \\
\hline Standard deviation & 42.92 & 1.70 & 64.03 & 3.20 & 109.90 & 6.74 \\
\hline \multicolumn{7}{|c|}{$\begin{array}{l}\text { Estimates from signal } \\
\text { at SNR of } 30 \mathrm{~dB}\end{array}$} \\
\hline Estimated mean & 102318.50 & 190.46 & 164977.18 & 323.86 & 250757.70 & 484.81 \\
\hline Standard deviation & 993.43 & 28.75 & 1520.14 & 44.89 & 2627.25 & 83.45 \\
\hline Actual value & 104920 & 202 & 168060 & 324 & 256150 & 494 \\
\hline
\end{tabular}

\subsection{Physical parameter identification of the nonlinear isolation layer}

The parameter identification of the nonlinear isolation system was performed via the proposed two-step procedure. Figure 9 shows the identified equivalent linear system stiffness and damping varied with the half cycle displacement increment. Compared with Figure 8, it is clear that the equivalent linear system stiffness decrease and damping increases at larger displacement increment half cycles, which indicates the isolation system softens as expected due to hysteretic nonlinear behaviour under the large portion of the ground motion excitation.

Figure 9 can be divided into a linear regime and a nonlinear regime. In the linear regime, the identified stiffness and damping parameter are nearly constant. It is difficult to accurately distinguish the linear and nonlinear regime. However, with some prior estimates and knowledge of the isolation system performance, such as by static or cyclic loading experiments, and observation of Figure 9, a probable transition phase from linear to nonlinear behaviour can be estimated. Herein, the half cycles with displacement increment between 1 to 
10mm are identified as linear half cycles. The half cycles with displacement increment exceeding 20mm are identified as nonlinear half cycles. These thresholds could be readily generated a priori to using the method from simulation and experimental analysis.
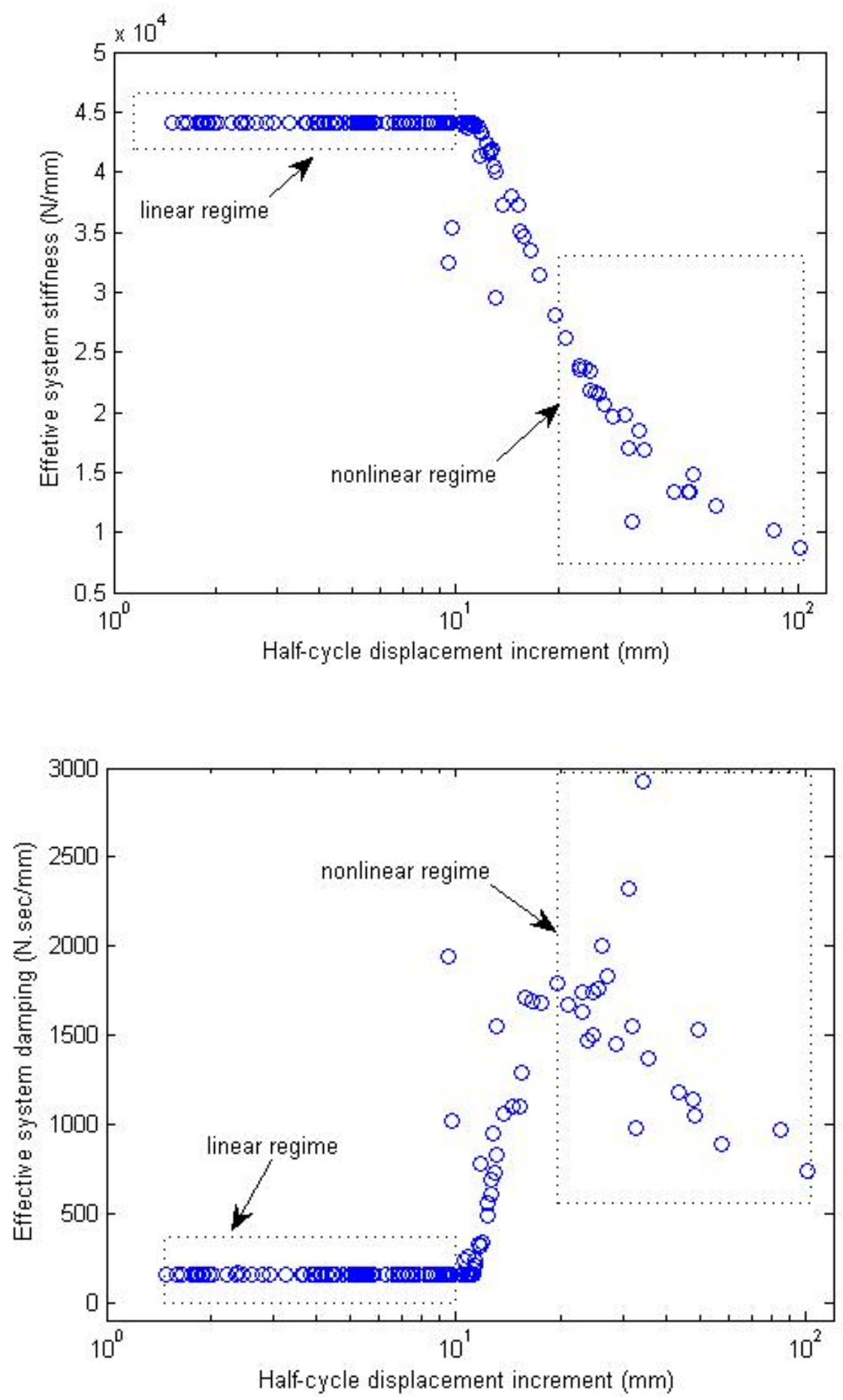

Figure 9 Effective linear system stiffness (top) and damping (bottom) identification results for the isolation system using noise-free signals 
For the first step, standard multiple linear regression is implemented to those identified linear half cycles to get estimates of the linear viscous damping coefficients $c_{0}$ and elastic stiffness $k_{\mathrm{e}}$ of the isolation layer. Table 2 shows the statistical results of identified parameters for different noise levels. It can be seen that the estimate accuracy of the stiffness parameter is better than the damping parameter. The identified viscous damping coefficients for different noise levels will be used as the known parameters in the next identification step.

Table 2 Linear viscous damping and elastic stiffness identification results for the isolation layer

\begin{tabular}{lcc}
\hline & $k_{\mathrm{e}}$ & $c_{0}$ \\
\hline Estimates from noise-free signal & 43888.10 & 188.71 \\
Estimated mean & & \\
Estimates from signal at SNR of $70 \mathrm{~dB}$ & 43888.11 & 188.71 \\
Estimated mean & 0.63 & 0.04 \\
Standard deviation & & 188.68 \\
Estimates from signal at SNR of $50 \mathrm{~dB}$ & 43888.57 & 0.42 \\
Estimated mean & 5.85 & 187.80 \\
Standard deviation & & 4.12 \\
Estimates from signal at SNR of $30 \mathrm{~dB}$ & 43897.84 & 156 \\
Estimated mean & 102.87 & \\
Standard deviation & & 44145 \\
\hline Actual value & & \\
\hline The unit for stiffness is N/mm and for damping is N.sec/mm & & \\
\hline
\end{tabular}

The unit for stiffness is $\mathrm{N} / \mathrm{mm}$ and for damping is N.sec/mm. 
Table 3 Nonlinear physical parameter identification results for the isolation system

\begin{tabular}{|c|c|c|c|}
\hline & $k_{\mathrm{e}}$ & $k_{\mathrm{p}}$ & $d_{\mathrm{y}}$ \\
\hline \multicolumn{4}{|c|}{ Estimates from noise-free signal } \\
\hline Estimated mean & 43187.99 & 7075.49 & 5.49 \\
\hline \multicolumn{4}{|c|}{ Estimates from signal at SNR of $70 \mathrm{~dB}$} \\
\hline Estimated mean & 43191.91 & 7076.29 & 5.49 \\
\hline Standard deviation & 11.20 & 3.03 & 0.00 \\
\hline \multicolumn{4}{|c|}{ Estimates from signal at SNR of $50 \mathrm{~dB}$} \\
\hline Estimated mean & 43195.27 & 7072.70 & 5.49 \\
\hline Standard deviation & 55.05 & 6.69 & 0.01 \\
\hline \multicolumn{4}{|c|}{ Estimates from signal at SNR of $30 \mathrm{~dB}$} \\
\hline Estimated mean & 42578.06 & 7080.74 & 5.56 \\
\hline Standard deviation & 171.26 & 14.83 & 0.03 \\
\hline Actual & 44145 & 6886 & 5.56 \\
\hline
\end{tabular}

The unit for stiffness is $\mathrm{N} / \mathrm{mm}$, for damping is $\mathrm{N} . \mathrm{sec} / \mathrm{mm}$ and for yield displacement is $\mathrm{mm}$.

For the second step, nonlinear regression analysis is implemented to those identified nonlinear half cycles to yield nonlinear physical parameters of the isolation system. Table 3 presents the statistical results of identified nonlinear physical parameters. It can be seen from Table 3 that the nonlinear regression analysis yields good performance to within $4 \%$ (worst case). As the noise level increases, the identification accuracy shows very little decrease. These latter results indicate the overall adequacy of the proposed two-step identification method for nonlinear multi-degree of freedom dynamic systems, as presented and in general.

It is noted that there is no direct comparative assessment of the proposed method against an existing methods. The primary reason is that no prior methods split the linear half-cycles from the nonlinear half-cycles of response and pull out nonlinear half-cycle displacement and post-yielding stiffness, except Nayerloo et al [42], which is a much more complex, but realtime, algorithm. Equally importantly, the work of Nayerloo et al [42] is restricted to fitting a Bouc-Wen model, whereas this approach is more general to any nonlinear, elasto-plastic 
method. Finally, it is important to note that we found no prior works that directly identified nonlinear stiffness in this fashion making direct comparison very difficult for those that do address nonlinear behaviour.

Although the efficiency of the method is demonstrated using a simple closed-formed problem, the value of the proposed method can be evaluated from three perspectives. First, the key of the method is to capture half-cycles and get elasto-plastic properties from them. It is not dependent on a specific mechanics model, but instead relies on direct measurements and identified half-cycles. Thus, the proposed method can be generalized to identify similar hysteretic systems, which nonlinear half cycle shape can be approximated by a bilinear shape. Second, the identification procedure is carried out from half-cycle to half cycle. It thus can capture time-variant physical parameters to characterize a degrading hysteretic system. Third, the identification procedure is essentially performed storey by storey. The identification method for the isolation layer can be applied to superstructure if nonlinearity needs to be considered for the superstructure. Therefore, the proposed method is completely generalizable to overall nonlinear multi-storey structures and a wide range of mechanics.

In addition, the approach can be extended to further portions of the half-cycles to identify further types of hysteresis loop. The schematic of Figure 2 is generic to a broad range of hysteresis loops, including the well-known Bouc-Wen model. However, extension to include pinching behaviours and further nonlinear mechanics requires only the addition of further regression steps to identify whether there are 3 or more segments in a given half-cycle, from which these more nonlinear behaviours and resulting nonlinear stiffness values could be reconstructed. Thus, this model-free method is generalisable to further, more complex nonlinear mechanics, although not shown specifically and is the subject of ongoing work. 


\section{CONCLUSIONS}

This paper presents a novel method, based on linear and nonlinear regression analysis techniques, for identification of the linear and nonlinear physical parameters of base-isolated multi-storey buildings using earthquake records. For the linear superstructure, a one-step multiple linear regression analysis is implemented to yield storey stiffness and damping parameters. For the nonlinear isolation layer, a two-step regression-based identification method is proposed. Nonlinear regression techniques are used to directly obtain elastic stiffness, post-yielding stiffness and yield displacement of the isolation system. A proof of concept case study has demonstrated the potential and feasibility of the proposed method. Identified system linear and nonlinear physical parameters are in very good agreement with those of the actual model values, even with a considerable level of measurement noise.

It is important to note this nonlinear identification procedure is simple and computationally straightforward. It can directly identify nonlinear isolation parameters without complex iterative optimization. The nonlinear regression algorithm requires no operator input to adjust optimization process, where previously proposed nonlinear output-error optimization methods have to select various weighting functions or control parameters to get optimal results. Although nonlinearity is considered only for the isolation system, the proposed method is completely generalizable to overall nonlinear multi-storey structures, where inelastic responses of both isolation system and the superstructure are allowed. Finally, the nonlinear regression algorithm is not only limited to bilinear hysteretic behaviour, tri-linear or more complex hysteretic models can also be identified by the proposed method with a little modification. For example, if we want to characterize the isolator with a tri-linear physical 
model, the nonlinear regression model used in the second step should be changed from a twophase linear regression function to a three-phase linear regression function.

Overall, the proposed method tested only for 2D shear-type framed structures and remains to be experimentally proven and further tested in more complex situations. However, it is a first step forward and can be readily generalized to 3D shear-type framed structures, as long as the 3D motion can be resolved. 


\section{ACKNOWLEDGMENT}

The present work was supported in part by China Scholarship Council for post-doctoral fellow (No.201203070008). The authors would like to thank the reviewers for their comments that help improve the manuscript. 


\section{REFERENCES}

1. Kelly, J.M., Aseismic base isolation: review and bibliography. Soil Dynamics and Earthquake Engineering, 1986. 5(4): p. 202-216.

2. Buckle, I.G. and R.L. Mayes, Seismic isolation: history, application, and performance-a world view. Earthquake spectra, 1990. 6(2): p. 161-201.

3. Kunde, M. and R. Jangid, Seismic behavior of isolated bridges: A-state-of-the-art review. Electronic Journal of Structural Engineering, 2003. 3(2): p. 140-169.

4. Warn, G.P. and K.L. Ryan, A Review of Seismic Isolation for Buildings: Historical Development and Research Needs. Buildings, 2012. 2(3): p. 300-325.

5. T E Kelly, R.I.S., W H Robinson, Seismic Isoaltion for Designers and Strucutre Engineers. 2007: Robinson Seismic Limited, New Zealand.

6. Stewart, J.P., J.P. Conte, and I.D. Aiken, Observed behavior of seismically isolated buildings. Journal of Structural Engineering, 1999. 125(9): p. 955-964.

7. Nagarajaiah, S. and S. Xiaohong, Response of base-isolated USC hospital building in Northridge earthquake. Journal of structural engineering, 2000. 126(10): p. 1177-1186.

8. Nagarajaiah, S. and X. Sun, Base-isolated FCC building: impact response in Northridge earthquake. Journal of Structural Engineering, 2001. 127(9): p. 1063-1075.

9. Nagarajaiah, S. and Z. Li, Time segmented least squares identification of base isolated buildings. Soil Dynamics and Earthquake Engineering, 2004. 24(8): p. 577-586.

10. Yoshimoto, R., A. Mita, and K. Okada, Damage detection of base - isolated buildings using multi - input multi - output subspace identification. Earthquake engineering \& structural dynamics, 2005. 34(3): p. 307-324.

11. Loh, C.H., et al., System identification of mid - story isolation building using both ambient and earthquake response data. Structural Control and Health Monitoring, 2013. 20(2): p. 1935.

12. Yao, R. and S.N. Pakzad, Time and frequency domain regression - based stiffness estimation and damage identification. Structural Control and Health Monitoring, 2013. 
13. Tan, R. and I. Weng, Identification of dynamic properties of isolated structures. Engineering structures, 1996. 18(3): p. 240-246.

14. Tan, R. and M. Huang, System identification of a bridge with lead-rubber bearings. Computers \& Structures, 2000. 74(3): p. 267-280.

15. Huang, M.C., et al., Physical system identification of an isolated bridge using seismic response data. Structural Control and Health Monitoring, 2009. 16(2): p. 241-265.

16. Huang, M.-C., et al., Physical-Parameter Identification of Base-Isolated Buildings Using Backbone Curves. Journal of structural engineering, 2009. 135(9): p. 1107-1114.

17. Huang, M.-C., et al., Development of physical-parameter identification procedure for in-situ buildings with sliding-type isolation system. Journal of Sound and Vibration, 2013. 332(13): p. $3315-3328$.

18. Furukawa, T., et al., System identification of base-isolated building using seismic response data. Journal of engineering mechanics, 2005. 131(3): p. 268-275.

19. Ahn, I.-S. and S.S. Chen, Nonlinear Model-Based System Identification of Lead-Rubber Bearings. Journal of structural engineering, 2008. 134(2): p. 318-328.

20. Xie, L. and A. Mita, Using component mode synthesis to estimate the restoring force of an isolation layer subjected to earthquakes. Structural Control and Health Monitoring, 2010. 17(2): p. 152-177.

21. Oliveto, N.D., G. Scalia, and G. Oliveto, Time domain identification of hybrid base isolation systems using free vibration tests. Earthquake Engineering \& Structural Dynamics, 2010. 39(9): p. 1015-1038.

22. Oliveto, G., et al. A preliminary study on the identification of seismic isolation systems from earthquake records. in 10CUEE Conference Proceedings.

23. Chen, B., Y. Xu, and X. Zhao, Integrated vibration control and health monitoring of building structures: a time-domain approach. Smart Structures and Systems, 2010. 6(7): p. 811-833.

24. Ueng, J.-M., C.-C. Lin, and P.-L. Lin, System identification of torsionally coupled buildings. Computers \& Structures, 2000. 74(6): p. 667-686. 
25. Gattulli, V. and F. Romeo, Integrated procedure for identification and control of MDOF structures. Journal of engineering mechanics, 2000. 126(7): p. 730-737.

26. Wu, M. and A. Smyth, Real-time parameter estimation for degrading and pinching hysteretic models. International Journal of Non-Linear Mechanics, 2008. 43(9): p. 822-833.

27. Smyth, A., et al., On-line parametric identification of MDOF nonlinear hysteretic systems. Journal of Engineering Mechanics, 1999. 125(2): p. 133-142.

28. Higashino, M. and S. Okamoto, Response control and seismic isolation of buildings. 2006: Taylor \& Francis.

29. Kelly, J.M. and D. Konstantinidis, Mechanics of rubber bearings for seismic and vibration isolation. 2011: John Wiley \& Sons.

30. Yan, X., Linear regression analysis: theory and computing. 2009: World Scientific.

31. Kelly, J.M., Earthquake-resistant design with rubber. 1993: Springer.

32. Gallant, A.R. and W.A. Fuller, Fitting segmented polynomial regression models whose join points have to be estimated. Journal of the American Statistical Association, 1973. 68(341): p. 144-147.

33. Lerman, P., Fitting segmented regression models by grid search. Applied Statistics, 1980: p. 77-84.

34. Cologne, J. and R. Sposto, Smooth piecewise linear regression splines with hyperbolic covariates. Journal of Applied Statistics, 1994. 21(4): p. 221-233.

35. Stoimenova, E., M. Datcheva, and T. Schanz, Application of two-phase regression to geotechnical data. Pliska Stud. Math. Bulgar, 2004. 16: p. 245-257.

36. Mata, J., Interpretation of concrete dam behaviour with artificial neural network and multiple linear regression models. Engineering Structures, 2011. 33(3): p. 903-910.

37. Skolnik, D.A. and J.W. Wallace, Critical assessment of interstory drift measurements. Journal of structural engineering, 2010. 136(12): p. 1574-1584.

38. Smyth, A. and M. Wu, Multi-rate Kalman filtering for the data fusion of displacement and acceleration response measurements in dynamic system monitoring. Mechanical Systems and Signal Processing, 2007. 21(2): p. 706-723. 
39. Hann, C.E., et al., Real-time system identification of a nonlinear four-story steel frame structure-Application to structural health monitoring. Sensors Journal, IEEE, 2009. 9(11): p. 1339-1346.

40. Hwang, J., et al., Optimal methods of RTK-GPS/accelerometer integration to monitor the displacement of structures. Sensors, 2012. 12(1): p. 1014-1034.

41. Hong, Y.H., S.G. Lee, and H.S. Lee, Design of the FEM-FIR filter for displacement reconstruction using accelerations and displacements measured at different sampling rates. Mechanical Systems and Signal Processing, 2013. 38(2): p. 460-481.

42. Nayyerloo, M., et al., LMS-based approach to structural health monitoring of nonlinear hysteretic structures. Structural Health Monitoring, 2011. 10(4): p. 429-444. 